\title{
THE FEKETE-SZEGÖ PROBLEM FOR STRONGLY CLOSE-TO-CONVEX FUNCTIONS
}

\author{
H. R. ABDEL-GAWAD AND D. K. THOMAS
}

(Communicated by Clifford J. Earle, Jr.)

\begin{abstract}
Let $K(\beta)$ denote the class of normalized analytic strongly close-toconvex functions of order $\beta \geq 0$, defined in the unit disc $D$ and let $f \in K(\beta)$, with $f(z)=z+a_{2} z^{2}+a_{3} z^{3}+\cdots$, for $z \in D$. Sharp bounds are obtained for $\left|a_{3}-\mu a_{2}^{2}\right|$ when $\mu$ is real.
\end{abstract}

\section{INTRODUCTION}

Denote by $S$ the class of normalized analytic univalent functions $f$ defined for $z \in D=\{z:|z|<1\}$ by

$$
f(z)=z+\sum_{n=2}^{\infty} a_{n} z^{n}
$$

A classical theorem of Fekete and Szegö [2] states that for $f \in S$ given by (1),

$$
\left|a_{3}-\mu a_{2}^{2}\right| \leq \begin{cases}3-4 \mu, & \text { if } \mu \leq 0 \\ 1+2 e^{-2 \mu /(1-\mu)}, & \text { if } 0 \leq \mu<1 \\ 4 \mu-3, & \text { if } \mu \geq 1 .\end{cases}
$$

This inequality is sharp in the sense that for each $\mu$ there exists a function in $S$ such that equality holds. Recently Pfluger [8] has considered the problem when $\mu$ is complex. In the case of $C, S^{*}$ and $K$, the subclasses of convex, starlike and close-to-convex functions respectively, the above inequalities can be improved [5,6]. In particular for $f \in K$ and given by (1), Keogh and Merkes [5] showed that

$$
\left|a_{3}-\mu a_{2}^{2}\right| \leq \begin{cases}3-4 \mu, & \text { if } \mu \leq 1 / 3, \\ 1 / 3+4 / 9 \mu, & \text { if } 1 / 3 \leq \mu \leq 2 / 3, \\ 1, & \text { if } 2 / 3 \leq \mu \leq 1, \\ 4 \mu-3, & \text { if } \mu \geq 1 .\end{cases}
$$

Again, for each $\mu$, there is a function in $K$ such that equality holds. In this paper we extend this result to the class $K(\beta)$ of strongly close-to-convex functions of order $\beta$ in the sense of Pommerenke [9]. Thus $f \in K(\beta)$ if, and

Received by the editors March 9, 1989.

1980 Mathematics Subject Classification (1985 Revision). Primary 30C45. 
only if, $f$, given by (1), is analytic in $D$ and is such that there exists $g \in S^{*}$ satisfying

$$
\left|\arg \frac{z f^{\prime}(z)}{g(z)}\right| \leq \frac{\pi \beta}{2}
$$

for $z \in D$ and $\beta \geq 0$. Clearly $K(0)=C, K(1)=K$ and when $0 \leq \beta \leq 1$, $K(\beta)$ is a subset of $K$ and hence contains only univalent functions. However in [4], Goodman showed that $K(\beta)$ can contain functions with unbounded valence for $\beta>1$.

Recently, Koepf [7] has considered the Fekete-Szegö problem for $K(\beta)$ and obtained sharp results for some particular values of $\mu$, all of which, with the exception of the case $\mu=1$ and $\beta \geq 1$, are contained in the following result.

\section{RESULTS}

Theorem. Let $f \in K(\beta)$ and be given by (1). Then for $0 \leq \beta \leq 1$,

$$
\left|a_{3}-\mu a_{2}^{2}\right| \leq \begin{cases}1-\mu+\frac{\beta(2-3 \mu)(\beta+2)}{3}, & \text { if } \mu \leq \frac{2 \beta}{3(\beta+1)}, \\ 1-\mu+\frac{2 \beta}{3}+\frac{\beta(2-3 \mu)^{2}}{3[2-\beta(2-3 \mu)]}, & \text { if } \frac{2 \beta}{3(\beta+1)} \leq \mu \leq \frac{2}{3}, \\ \frac{2 \beta+1}{3}, & \text { if } \frac{2}{3} \leq \mu \leq \frac{2(\beta+2)}{3(\beta+1)}, \\ \mu-1+\frac{\beta(3 \mu-2)(\beta+2)}{3}, & \text { if } \mu \geq \frac{2(\beta+2)}{3(\beta+1)},\end{cases}
$$

whilst for $\beta>1$, the first two inequalities hold. For each $\mu$ there are functions in $K(\beta)$ such that equality holds in all cases.

We shall require the following:

Lemma 1 ([10, p. 166]). Let $h \in P$, i.e., let $h$ be analytic in $D$ and satisfy $\operatorname{Re} h(z)>0$ for $z \in D$, with $h(z)=1+c_{1} z+c_{2} z^{2}+\cdots$, then

$$
\left|c_{2}-\frac{c_{1}^{2}}{2}\right| \leq 2-\frac{\left|c_{1}^{2}\right|}{2} \text {. }
$$

Lemma 2 ([6, Lemma 3]). Let $g \in S^{*}$ with $g(z)=z+b_{2} z^{2}+b_{3} z^{3}+\cdots$, then for $\mu$ real,

$$
\left|b_{3}-\mu b_{2}^{2}\right| \leq \max \{1,|3-4 \mu|\} .
$$

We note that Lemma 2 above can easily be extended to the wider class $S^{*}(\alpha)$ of strongly starlike functions of order $\alpha \geq 0$, i.e., $g$ analytic and normalized in $D$ and satisfying

$$
\left|\arg \frac{z g^{\prime}(z)}{g(z)}\right| \leq \frac{\alpha \pi}{2},
$$

see e.g. [1]. In this case, one obtains the sharp inequality

$$
\left|b_{3}-\mu b_{2}^{2}\right| \leq \max \left\{\alpha, \alpha^{2}|3-4 \mu|\right\},
$$

for $\mu$ real.

Proof of Theorem. It follows from (2) that we can write

$$
z f^{\prime}(z)=g(z) h(z)^{\beta}
$$


for $g \in S^{*}$ and $h \in P$. Equating coefficients in (3) we obtain $2 a_{2}=\beta c_{1}+b_{2}$ and $3 a_{3}=(\beta(\beta-1) / 2) c_{1}^{2}+\beta c_{2}+\beta c_{1} b_{2}+b_{3}$, so that

$$
\begin{aligned}
a_{3}-\mu a_{2}^{2}= & \frac{1}{3}\left(b_{3}-\frac{3}{4} \mu b_{2}^{2}\right)+\frac{\beta}{3}\left(c_{2}+\left(\frac{\beta(2-3 \mu)}{4}-\frac{1}{2}\right) c_{1}^{2}\right) \\
& +\beta\left(\frac{1}{3}-\frac{\mu}{2}\right) c_{1} b_{2} .
\end{aligned}
$$

We consider first the case $(2 \beta) /(3(\beta+1)) \leq \mu \leq 2 / 3$. Equation (4) gives

$$
\begin{aligned}
\left|a_{3}-\mu a_{2}^{2}\right| & \leq \frac{1}{3}\left|b_{3}-\frac{3}{4} \mu b_{2}^{2}\right|+\frac{\beta}{3}\left|c_{2}-\frac{1}{2} c_{1}^{2}\right|+\frac{\beta^{2}(2-3 \mu)}{12}\left|c_{1}^{2}\right|+\beta\left(\frac{1}{3}-\frac{\mu}{2}\right)\left|c_{1}\right|\left|b_{2}\right|, \\
& \leq 1-\mu+\frac{\beta}{3}\left(2-\frac{1}{2}\left|c_{1}^{2}\right|\right)+\frac{\beta^{2}(2-3 \mu)}{12}\left|c_{1}^{2}\right|+\frac{\beta(2-3 \mu)}{3}\left|c_{1}\right|, \\
& =\Phi(x) \text { say, with } x=\left|c_{1}\right|,
\end{aligned}
$$

where we have used Lemmas 1 and 2 and the fact that $\left|b_{2}\right| \leq 2$ for $g \in S^{*}$. An elementary argument shows that the function $\Phi$ attains a maximum at $x_{0}=2(2-3 \mu) /(2-\beta(2-3 \mu))$, and so

$$
\left|a_{3}-\mu a_{2}^{2}\right| \leq \Phi\left(x_{0}\right),
$$

which proves the theorem if $\mu \leq 2 / 3$ and $\beta \geq 0$. Choosing

$$
c_{1}=\frac{2(2-3 \mu)}{2-\beta(2-3 \mu)}, \quad c_{2}=2, \quad b_{2}=2, \quad \text { and } \quad b_{3}=3,
$$

in (4) shows that the result is sharp. We note that $\left|c_{1}\right| \leq 2$, that is, $\mu \geq$ $2 \beta /(3(\beta+1))$.

Next consider the case $\mu \leq(2 \beta) /(3(\beta+1))$. Then

$$
\begin{aligned}
\left|a_{3}-\mu a_{2}^{2}\right| & \leq\left|a_{3}-\frac{2 \beta}{3(\beta+1)} a_{2}^{2}\right|+\left(\frac{2 \beta}{3(\beta+1)}-\mu\right)\left|a_{2}\right|^{2}, \\
& \leq 1+\frac{2 \beta}{3}+\left(\frac{2 \beta}{3(\beta+1)}-\mu\right)(\beta+1)^{2}=1-\mu+\frac{\beta(2-3 \mu)(\beta+2)}{3},
\end{aligned}
$$

for $\beta \geq 0$, where we have used the result already proved in the case $\mu=$ $2 \beta / 3(\beta+1)$, and the fact that for $f \in K(\beta)$, the inequality $\left|a_{2}\right| \leq \beta+1$ holds [3]. Equality is attained on choosing $\lambda=0, p_{1}=p_{2}=b_{2}=2$, and $b_{3}=3$.

Suppose now that $2 / 3 \leq \mu \leq(2(\beta+2)) /(3(\beta+1))$. Since $g \in S^{*}$ we can write $z g^{\prime}(z)=g(z) p(z)$ for $p \in P$, with $p(z)=1+p_{1} z+p_{2} z^{2}+\cdots$, and so equating coefficients we have that $b_{2}=p_{1}$ and $2 b_{3}=p_{1}^{2}+p_{2}$.

We deal first with the case $\mu=2(\beta+2) /(3(\beta+1))$. Thus (4) gives

$$
\begin{aligned}
a_{3}-\frac{2(\beta+2)}{3(\beta+1)} a_{2}^{2}= & \frac{1}{6}\left(p_{2}-\frac{p_{1}^{2}}{2}\right)+\frac{\beta}{3}\left(c_{2}-\frac{c_{1}^{2}}{2}\right)+\frac{\beta-1}{12(\beta+1)} p_{1}^{2} \\
& -\frac{\beta^{2} c_{1}^{2}}{6(\beta+1)}-\frac{\beta p_{1} c_{1}}{3(\beta+1)}
\end{aligned}
$$


and so if $\beta \leq 1$,

$$
\begin{aligned}
\left|a_{3}-\frac{2(\beta+2)}{3(\beta+1)} a_{2}^{2}\right| \leq & \frac{1}{6}\left|p_{2}-\frac{p_{1}^{2}}{2}\right|+\frac{\beta}{3}\left|c_{2}-\frac{c_{1}^{2}}{2}\right|+\frac{(1-\beta)}{12(\beta+1)}\left|p_{1}^{2}\right| \\
& +\frac{\beta^{2}\left|c_{1}^{2}\right|}{6(\beta+1)}+\frac{\beta\left|p_{1} c_{1}\right|}{3(\beta+1)}, \\
\leq & \frac{1}{6}\left(2-\frac{\left|p_{1}^{2}\right|}{2}\right)+\frac{\beta}{3}\left(2-\frac{\left|c_{1}^{2}\right|}{2}\right)+\frac{1-\beta}{12(\beta+1)}\left|p_{1}^{2}\right| \\
& +\frac{\beta^{2}\left|c_{1}^{2}\right|}{6(\beta+1)}+\frac{\beta\left|p_{1} c_{1}\right|}{3(\beta+1)} \\
= & \frac{2 \beta+1}{3}-\frac{\beta}{6(\beta+1)}\left(\left|c_{1}\right|-\left|p_{1}\right|\right)^{2}, \\
\leq & \frac{2 \beta+1}{3},
\end{aligned}
$$

where we have used Lemma 1.

Now write

$$
\begin{aligned}
a_{3}-\mu a_{2}^{2}= & \frac{(\beta+1)(3 \mu-2)}{2}\left(a_{3}-\frac{2(\beta+2)}{3(\beta+1)} a_{2}^{2}\right) \\
& +\frac{3(\beta+1)}{2}\left(\frac{2(\beta+2)}{3(\beta+1)}-\mu\right)\left(a_{3}-\frac{2}{3} a_{2}^{2}\right),
\end{aligned}
$$

and the result follows at once on using the theorem already proved in the cases $\mu=2 / 3$ and $\mu=2(\beta+2) /(3(\beta+1))$ for $\beta \leq 1$. Equality is attained when $f$ is given by

$$
f^{\prime}(z)=\frac{\left(1+z^{2}\right)^{\beta}}{\left(1-z^{2}\right)^{\beta+1}} .
$$

We finally assume that $\mu \geq(2(\beta+2)) /(3(\beta+1))$. Write

$$
a_{3}-\mu a_{2}^{2}=\left(a_{3}-\frac{2(\beta+2)}{3(\beta+1)} a_{2}^{2}\right)+\left(\frac{2(\beta+2)}{3(\beta+1)}-\mu\right) a_{2}^{2},
$$

and the result follows at once on using the theorem already proved for $\mu=$ $2(\beta+2) / 3(\beta+1))$ in the case $\beta \leq 1$ and the inequality $\left|a_{2}\right| \leq \beta+1$, which was proved in [3]. Equality is attained in this last case on choosing $c_{1}=b_{2}=2 i$, $c_{2}=-2$ and $b_{3}=-3$ in (4).

We remark that the methods used in $[5,6]$, together with equation $(4)$, suggest that in order to obtain sharp results for $\beta>1$ and $\mu>2 / 3$, an extension to the "area principle" may be required. Since $K(\beta)$ contains functions of unbounded valence for $\beta>1$ establishing sharp estimates in this case may require deeper methods.

\section{REFERENCES}

1. D. A. Brannan and W. E. Kirwan, On some classes of bounded univalent functions, J. London Math. Soc. (2) 1 (1969), 431-443.

2. M. Fekete and G. Szegö, Eine Bermerkung über ungerade schlichte Funktionen, J. London Math. Soc. 8 (1933), 85-89.

3. A. W. Goodman, On close-to-convex functions of higher order, Ann. Univ. Sci. Budapest. Eötvös Sect. Math. 15 (1972), 17-30. 
4. _ A note on the Noshiro-Warschawski theorem, J. Analyse Math. 25 (1972), 401-408.

5. F. R. Keogh and E. P. Merkes, A coefficient inequality for certain classes of analytic functions, Proc. Amer. Math. Soc. 20 (1969), 8-12.

6. W. Koepf, On the Fekete-Szegö problem for close-to-convex functions, Proc. Amer. Math. Soc. 101 (1987), 89-95.

7. 420-433.

8. A. Pfluger, The Fekete-Szegö inequality for complex parameters, Complex Variables Theory Appl. 7 (1986), 149-160.

9. Ch. Pommerenke, On close-to-convex analytic functions, Trans. Amer. Math. Soc. 114 (1965), 176-186.

10. _ Univalent functions, Vandenhoeck and Ruprecht, Göttingen, 1975.

Department of Mathematics and Computer Science, University College Swansea, SWANSEA SA2 8PP, WALES 